hältnifs $\frac{\alpha}{\gamma}<\sqrt{\frac{1}{2}}$, mit welehem sich das flüssige Sphäroid mit permanenter Figur um die kürzeste Axe drehen kann."

4. Ich will jetzt noch untersuchen, ob das Gleichgewicht möglich ist, wenn sich das Sphäroid um die mittlere Axe dreht. Für diesen Fall werden die Gleichungen (6)

$$
\left.\begin{array}{l}
\frac{\mu}{\alpha \alpha}=\frac{3 M}{\gamma^{3} \sin \varphi^{3}} \int \frac{\operatorname{tg} \phi^{2} \cdot d \varphi}{\sqrt{\left(1-e^{2} \sin \phi^{2}\right)}}-n n \\
\frac{\mu}{\beta \beta}=\frac{3 M}{\gamma^{3} \sin \varphi^{3}} \int \frac{\sin \phi^{2} \cdot d \varphi}{\left(1-e^{2} \sin \phi^{2}\right)^{\frac{3}{2}}} \\
\frac{\mu}{\gamma \gamma}=\frac{3 M}{\gamma^{3} \sin \varphi^{3}} \int \frac{\sin \phi^{2} \cdot d \varphi}{\sqrt{\left(1-e^{2} \sin \phi^{2}\right)}-n n}
\end{array}\right\}
$$

Eliminirt man wieder $\mu$ und $n n$ aus diesen Gleichungen, so ergiebt sich nach Substitution von $\alpha$ und $\beta$ folgende Bedin. gungsgleichung:

$$
\begin{aligned}
& 0=\left(1-e^{2} \sin \phi^{2}\right) \sin \varphi^{2} \int \frac{\sin \phi^{2} d \varphi}{\left(1-e^{2} \sin \varphi^{2}\right)^{\frac{3}{2}}}-\cos \varphi^{2} \int \frac{\sin \varphi^{4} d \varphi}{\cos \phi^{2} \sqrt{\left(1-e^{2} \sin \varphi^{2}\right)}}=W, \\
& \left(1-2 e^{2} \sin \varphi^{2}\right) \int \frac{\sin \varphi^{2} d \varphi}{\left(1-e^{2} \sin \phi^{2}\right)^{\frac{3}{2}}}+\int \frac{\sin \varphi^{4} d \varphi}{\cos \phi^{2} V\left(1-e^{2} \sin \phi^{2}\right)}=\frac{d W_{1}}{2 \sin \phi \cos \phi d \phi} \\
& \left(\left(1-e^{2} \sin \varphi^{2}\right)^{2}+e^{2}\left(1-e^{2}\right) \sin \varphi^{4}\right) \int \frac{\sin \phi^{2} d \varphi}{\left(1-e^{2} \sin \phi^{2}\right)^{\frac{3}{2}}}=W_{1}+\frac{\cos \varphi d W_{1}}{2 \sin \phi d \phi}
\end{aligned}
$$

Wenn eine der Grölsen $\mathscr{W}$, und $d W$, verschwindet, ist die andere immer positiv, da $\left(1-e^{2} \sin \varphi^{2}\right)^{2}+e^{2}\left(1-e^{2}\right) \sin \varphi^{4}$ positiv ist. Es ist $d W$, positiv von $\varphi=0^{\circ}$ bis $\sin \varphi=\frac{1}{e \sqrt{2}}$. Ist demnach der kleinste Werth von $\varphi$, für welchen $W,=0$, so wird $d W_{1}$, da $W$, von positiv in negativ übergeht, für diesen Werth von $\varphi$ negativ, im Widerspruch mit der letzten Gleichung. Das Sphäroid kann sich demnach nicht um die mittlere Axe drehen, wenn die flüssige Masse in Gleichgewicht gehalten werden soll.

5. Nimmt man an, das Sphäroid drehe sich um die läng-

ste Axe, so werden die Bedingungsgleichungen für den Fall des Gleichgewichts

$$
\left.\begin{array}{rl}
\frac{\mu}{\alpha \alpha} & =\frac{3 M}{\gamma^{3} \sin \varphi^{3}} \int \frac{\operatorname{tg} \varphi^{2} d \varphi}{\sqrt{\left(1-e^{2} \sin \phi^{2}\right)}}-n n \\
\frac{\mu}{\beta \beta} & =\frac{3 M}{\gamma^{3} \sin \varphi^{3}} \int \frac{\sin \phi^{2} d \varphi}{\left(1-e^{2} \sin \phi^{2}\right)^{\frac{3}{2}}}-n n \\
\frac{\mu}{\gamma \gamma} & =\frac{3 M}{\gamma^{3} \sin \varphi^{3}} \int \frac{\sin \phi^{2}}{\left.\sqrt{\left(1-e^{2}\right.} \sin \phi^{2}\right)}
\end{array}\right\} \ldots . .
$$

Eliminirt man wiederum $\mu$ und $n n$, so findet man nach Sub. stitution von $\alpha$ und $\beta$ die Bedingungsgleichung

$$
\begin{aligned}
& 0=\left(1-e^{2} \sin \varphi^{2}\right) \cos \phi^{2} \int \frac{\sin \phi^{4} d \varphi}{\cos \varphi^{2}\left(1-e^{2} \sin \varphi^{2}\right)^{\frac{3}{2}}}-\sin \phi^{2} \int \frac{\sin \phi^{2} d \varphi}{\sqrt{\left(1-e^{2} \sin \varphi^{2}\right)}}=W_{\prime \prime}
\end{aligned}
$$

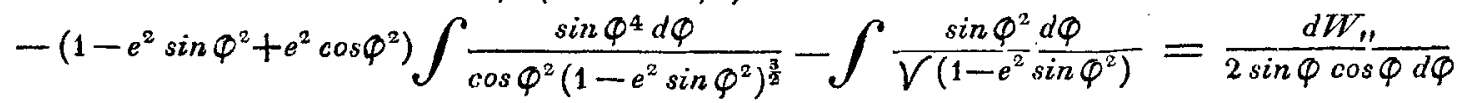

Da $d W_{\prime \prime}$ von $\varphi=0$ bis $\varphi=\frac{\pi}{2}$ beständig negativ ist, und $W_{1 \prime}=0$ für $\varphi=0$; so kann $W_{n}$ nicht $=0$ werden innerhalb dieser Grenzen $\varphi=0$ ausgeschlossen.

Es kann demnach das Gleichgewicht nur Statt finden, weno die flüssige Masse sich um die kürzeste Axe dreht.

Th. Clausen.

\title{
Beobachtungen von Sonnenflecken im Jahre 1840.
}

\section{Vou Herrn Hofrath Schwabe.}

In diesem Jahre konnte ich die Sonne an 263 Tagen beob. achten und 152 Fleckengruppen zählen. Am 15 $15^{\text {ten }}, 16^{\text {ten }}$ und $17^{\text {ten }}$ April fand ich sie vollkommen fleckenlos und in der Zone, worin die Flecken entstehen, zeigten sich starke Narben und einige geballte Lichtwolken, worin ich mit 64 und 96maliger Vergröfserung des 6füls. Fraunhoferschen Fernrohrs nur sehr feine Poren entdeckte. Die wenigsten Flecke hatte die Sonne am $7^{\text {ten }}$ und $8^{\text {ten }}$ Decbr., indem nur einige feine, jedoch schon mit dem $3 \frac{1}{2}$ füfs. Fraunhofer 42 und $84 \mathrm{mal}$. Vergrölserung bemerkbaren Punkte sichtbar waren. Vorzüglich grofse Ansamm. lungen von Fleckengruppen erzeugten sich nicht; die meisten
Gruppen waren am $1^{\text {sten }}$ und $2^{\text {ten }}$ September vorhanden und dennoch waren zu gleicher Zeit nur sieben sichtbar, worunter sich eine einzige von ausgezeichneter Gröfse befand. Die gröfsten und reichsten Gruppen dieses Jahres waren vom $19^{\text {ten }}$ bis $27^{\text {ten }}$ April, vom $1^{\text {sten }}$ bis $2^{\text {ten }}$ Mai, vom $12^{\text {ten }}$ bis $19^{\text {ten }}$ Juli, vom $19^{\text {ten }}$ bis $24^{\text {sten }}$ August, vom $1^{\text {sten }}$ bis $5^{\text {ten }}$ September, $17^{\text {ten }}$ bis $28^{\text {sten }}$ October, vom $1^{\text {sten }}$ bis $6^{\text {ten }}$ November und vom $12^{\text {ten }}$ bis $18^{\text {ten }}$ November sichtbar. Am merkwürdigste n war die öftere Wiederkehr dreier Gruppen, von denen die erste 2 Rotationen, die zweite 4 Rotationen und die dritte 8 Rotationen der Sonne aushielt. In den zwei ersten Gruppen war 10 * 
kein beständiger Flecken bemerkbar, die Gruppen traten nur zu den gehörigen Zeiten und an denselben Orten wieder eiv. Die dritte Gruppe zeigte aber, ungeachtet ihrer Veränderlichkeit, einen beständigen Flecken, der zwar an Gestalt und Grörse, nicht aber im Orte wechselte und 9mal am östlichen Sonnenrande eintrat. Ich füge ein Verzeichnifs bei, welches die Eintritte und Austritte so wie die Zeit enthült, als der Flecken bei jeder Revolution sich ungefähr in der Mitte seiner Bahn befand.

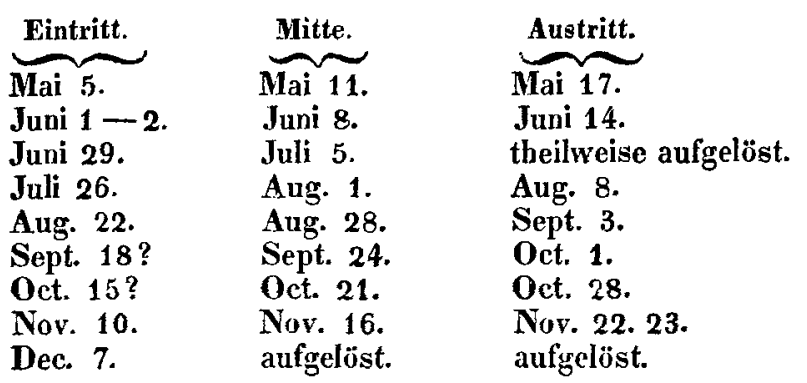

In den zwei ersten Vorübergängen war dieser Flecken klein, ohne Nebel und Hof und wurde von ähnlichen etwas kleinern und einigen Punkten begleitet. Beim dritten Vorübergang, wo er den 29sten Juni eintrat, war er nicht wesentlich verändert, löste sich aber bald theilweise auf, so dafs ich seinen Austritt nicht genau anzugeben im Stande war; jedoch trat er am 26sten Juli als ein behofter Kernllecken regelmäfsig ein, und sowohl er als die zu ihm gehörige Gruppe bildeten sich zu einer ansehulichen Grölse aus. Bei seinem fünften Ein- tritte am $22^{\text {sten }}$ August war er nur noch allein vorhanden und änderte sich auch während seines Vorüberganges nicht wesentlich. Am $18^{\text {ten }}$ September konnte ich seinen Eintritt wegen des bedeckten Himmels nicht bcobachten, fand ihn aber am

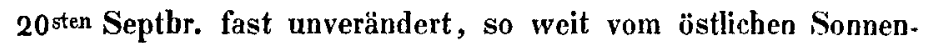
rande entfernt, dafs er am $18^{\text {ten }}$ eingetreten seyn mufste; dasselbe Ereignifs fand auch bei seinem $6^{\text {ten }}$ Vorübergang statt, wo ich ihn erst am $17^{\text {ten }}$ October sehen konnte, er war von mehreren andern Flecken und Punkten umgeben. Von nun an löste sich die Gruppe immer mehr auf, bis ich den behoften Kernflecken zuletzt am $22^{\text {sten }}$ November nahe am Westrande der Sonne allein ohne alle Nebenflecke beobachtete. Am 24sten war er ausgetreten. Sein neunter Eintritt erfolgte am $\gamma^{\text {ten }}$ December, jedoch war nur sehr helles geballtes Lichtgewölk an seiner Stelle sichtbar, worin ich erst am $9^{\text {ten }}$ mit dem $3 \frac{1}{2} \mathrm{f}$. 42 und 84mal. Vergröfserung einige feine Punkte erkennen konnte. Wenn man nun noch weiter zurückrechnet und die RevolutionsPeriode für diesen Flecken zu ungefähr $27 \mathrm{t}$ annimmt, erhält man für die Mitte seiner Bahn: April 13, März 17. Februar 1840 bleibt er aus. 1839 den $1^{\text {sten }}$ Dec., den $4^{\text {ten Nov., dev }}$ 9 ten Oct., den $12^{\text {ten }}$ Sept. tritt er regelmäfsig in die Mitte ein. Den $16^{\text {ten }}$ Aug. und den 20 sten Juli bleibt er aus, steht aber den $23^{\text {sten }}$ Juni wieder in der Mitte, bleibt den $27^{\text {sten }}$ Mai und $3^{\text {sten }}$ April aus, ist am $3^{\text {ten }}$ April in der Mitte sichtbar, den $7^{\text {ten }}$ März und $8^{\text {ten }}$ Febr. unsichtbar und den $12^{\text {ten }}$ Jan. wieder gegenwärtig. Die bekannten Lichtflocken bei der Sonne habe ich in diesem Jahre nicht gesehen.

Schwabe.

\section{Beobachtungen von Sonnenflecken auf der Altonaer Sternwarte. Von Herrn Observator Petersen.}

Die hier folgenden Beobachtungen von Sonnenflecken sind mit einem Fraunhofer von 27 Linien Oeffnung, 30 Zoll Brennweite und 60mal. Vergrörserung gemacht, der parallactisch aufgestellt und mit einem Troughtonschen Filarmicrometer versehen ist. Die Abstände der Flecken vem nördlichen und südlichen Sonnen rande sind durch Messungen mit diesem Micrometer ermittelt und in Bogen verwandelt in den Beobachtungen durch (S) Sïdr rand + gemessener Abstand, (N) Nordrand - gemessener Abstand notirt; später ist jedoch der Kürze wegen nur das Mittel aus beiden, oder der Abstand vom Mittelpuncte der Sonne selbst angegeben, wobei ein nördlicher Abstand der Flecken durch + , ein südlicher durch - hezeichnet ist. Der Rectascensions-Unterschied zwischen dem Flecken und dem ersten und zweiten Sonnenrande ist aus Fäden-Durchgängen an Fäden, welche im Micrometer senkrecht auf der täglichenBewegung gestellt waren, geschlossen und durch (I) erster Rand + Unterschied in
Zeit, (II) zweiter Rand - Unterschied in Zeit angegeben. Auch hier ist später nur das Mittel aus beiden, oder cler Zeitunterschied zwischen dem Mittelpuncte der Sonne und dem Flecken notirt, wo + einen östlichen, - einen westlichen Abstand des Fleckens vom Mittelpuncte der Sonne bezeichnet. Alle Beobachtungen sind von dem Einflusse der Strahlenbrechung befreit, die Parallaxe ist vernachlässigt. In den Rectascensionsbeobachtungen sind fast ohne Ausnahme bei jedem Fäden-Durchgang beide Sonnenränder beobachtet, hingegeu habe ich an einzelnen Tagen für die Declinations-Bestimmung nicht den Abstand aller Flecken von beiden Sonneurändern messen können, in welchen wenigen Fällen ( sie sind durch : bezeichnet) stets zur Ermittelung des Declinations-Unterschiedes der aus den übrigen Beobachtungen ich ergebende Sonnendurchmessers angewandt ist, welcher fast immer etwas gröfser gefunden wurde, als im Berliner Astron. Jahrbuche. 